\section{Book case history}

AlexanderG. Bearn

Companion Encyclopaedia of the History of Medicine. Edited by W. F. Bynum and Roy Porter. Routledge: 1993. Pp. Volume 1, 778; Volume 2, 997. £150, \$199.95.

THE dangers of editing an encyclopaedia, even a small one, are formidable. It took Diderot 20 years and many volumes to complete his Encyclopédie and his coeditor D'Alembert gave up in despair 15 years before its publication in 1772 . The Encyclopaedia Britannica, devised by a "society of gentlemen in Scotland", was completed in three volumes in 1771 and is still with us; the latest edition in 1992 running to 32 volumes.

W. F. Bynum and Roy Porter call these two volumes a "companion encyclopaedia". Avowedly neither comprehensive nor encyclopaedic, their work fits neatly between the genre of the typical 'encyclopaedia' and that of 'collected essays'. Its encyclopaedic dimension consists rather in the number of essays collected than in the comprehensiveness of its coverage. The Companion Encyclopaedia of the History of Medicine is a fascinating miscellany of 72 essays of varying length written by 36 carefully selected scholars, mostly British or American, in the history of medicine.

In an important introductory essay entitled "The Art of Science and Medicine", the editors have interpreted their subject in its evolving historical, social and cultural contexts. It is this broad sweep of medical history that sets this encyclopaedia apart from others in the field.

It is divided into seven somewhat arbitrary parts varying from the ambitiously titled Theories of Life, Health and Disease to more narrowly focused chapters on clinical medicine. The section on body systems includes succinct historical essays on basic disciplines such as anatomy, physiology, biochemistry and immunology that are important scientific platforms on which medicine necessarily rests.

An essay on the doctor-patient relationship opens the section on clinical medicine and contains one chapter in which Robert C. Olby considers the constitutionality of disease in enlightening and judicious prose. Christopher C. Booth provides an admirable essay on the role of clinical research in understanding the nature of human disease.

Essays on Arab Islamic medicine, Chinese medicine and Indian medicine are a particularly welcome and illuminating corrective to the more usual emphasis on Western culture.

In one of the more difficult essays, Arthur Caplan struggles with the distinction between health and disease. Although such a distinction may be a pragmatic necessity, particularly in the social context of governed allocation of limited resources, the concept is biologically unsatisfying. The view frequently expressed by René Dubos, particularly in his book The Mirage of Health, that complete freedom from disease is almost incompatible with the process of living, seems more rewarding.

The scholarly essay on psychotherapy by Sander Gilman outlines its chequered history. The ranks of those who cling to orthodox Freudian psychotherapy have steadily shrunk and the view expressed by Peter Medawar more than 20 years ago that "psychoanalysis will remain for ever one of the saddest and strangest landmarks in the history of twentieth-century thought" has more adherents.

The history of contagion and infectious diseases, discussed in more than one essay, is traced with erudition through the centuries. The overall decline in mortality from infectious disease over the past hundred years has been impressive. In his chapter on demography, R. M. Smith challenges T. McKeoun's view that this decline has been almost exclusively due to improvement in the environment on the grounds that he largely ignored demographic variations in mortality.

Microbes, old and new, remain formidable agents of disease. Tuberculosis has returned and been made more deadly by strains resistant to all known antibiotics. It is estimated that AIDS, unknown 15 years ago, will affect 40 million people by the end of the decade and is already firmly entrenched in the Indian subcontinent, South-East Asia and China, which comprise half the world's population. Almost a century has passed since Patrick Manson identified the anopheles mosquito as the transmitting agent for malaria. Malaria still kills 3,000 children a day in Africa. Airport malaria, particularly among baggage handlers, is well documented and improvements in airline schedules have enabled mosquitoes to become world travellers. Diseases previously regarded as geographically confined have become global. Only smallpox appears to have been eradicated.

The diversity of subjects not ordinarily considered in books of this nature hospitals, women and medicine, nursing, architecture, literature and medicine, medical philanthropy, to give only a few examples - make this not only a valuable and lively work of reference, but a book to

Aspects of medical history - The Surgeon by Jan Sanders van Hemessen (c.1504-66).

be kept close at hand and read with pleasure as well as enlightenment. For those who wish to extend their knowledge, the editors and authors have provided well chosen selected references. This encyclopaedia is an invaluable and scholarly source and deserves to be widely read by those interested in the broader aspects of medical history.

"All sciences", the historian C. V. Wedgwood (in her collected essays, History and Hope, 1987) reminds us, "are devoted to the quest for truth; truth can be neither apprehended nor communicated without art. History therefore is an art, like all the other sciences." This well indexed encyclopaedia, edited by two of the leading English scholars in the field, will inform and stimulate all those interested in the art and science of medical history.

Alexander G. Bearn is at The Rockefeller University, 1230 New York Avenue, New York 10021-6394, USA. 